\title{
Synthesis and reactions of some sulfur ylide complexes of palladium
}

\author{
M.C. Cheng, S.M. Peng ${ }^{\star}$ \\ Department of Chemistry, National Taiwan University, Taipei 10764 (Taiwan) \\ I.J.B. Lin ${ }^{\star}$, B.H.H. Meng and C.H. Liu \\ Department of Chemistry, Fu Jen Catholic University, Hsin Chuang 24205 (Taiwan)
}

(Received December 27th, 1986)

\begin{abstract}
The iodo-bridged sulfur ylide complex $\left[\mathrm{Pd}(\mu-\mathrm{I})\left(\left(\mathrm{CH}_{2}\right)_{2}(\mathrm{SO})\left(\mathrm{CH}_{3}\right)\right)\right]_{2}$ (1) when treated with dithiolates, acetylacetone and various Lewis bases gave $\left[\operatorname{Pd}\left(\left(\mathrm{CH}_{2}\right)_{2}-\right.\right.$ $\left.\left.(\mathrm{SO})\left(\mathrm{CH}_{3}\right)\right)(\mathrm{S} \sim \mathrm{S})\right] \quad\left(\mathrm{S} \sim \mathrm{S}=\mathrm{S}_{2} \mathrm{CN}\left(\mathrm{C}_{2} \mathrm{H}_{5}\right)_{2}, \quad \mathrm{~S}_{2} \mathrm{COC}_{2} \mathrm{H}_{5}\right.$ and $\left.\mathrm{S} 2 \mathrm{P}\left(\mathrm{OC}_{2} \mathrm{H}_{5}\right)_{2}\right)$, $\left[\mathrm{Pd}\left(\left(\mathrm{CH}_{2}\right)_{2}(\mathrm{SO})\left(\mathrm{CH}_{3}\right)\right)(\mathrm{acac})\right] \quad($ acac $=$ acetylacetonate $)$ and $\left[\mathrm{PdI}\left(\left(\mathrm{CH}_{2}\right)_{2}(\mathrm{SO})-\right.\right.$ $\left.\left(\mathrm{CH}_{3}\right)\right)($ base $\left.)\right]\left(\right.$ base $=\mathrm{PPh}_{3}, \mathrm{P}(\mathrm{OMe})_{3}, \mathrm{P}(\mathrm{OPh})_{3}$ and $\left.\mathrm{C}_{5} \mathrm{H}_{5} \mathrm{~N}\right)$. In the presence of a phase transfer catalyst (PTC), the reaction rates and yields were greatly increased. Reaction of several related sulfur ylide complexes with $\mathrm{I}_{2}, \mathrm{HI}$ or aqueous $\mathrm{NaOH}$ gave 1. The single crystal structure of $\left[\mathrm{Pd}\left(\left(\mathrm{CH}_{2}\right)_{2}(\mathrm{SO})\left(\mathrm{CH}_{3}\right)\right)_{2}\right]$ was determined (orthorhombic, Pbcn, a 13.379(2), b 8.081(1), $c$ 9.048(2) $\AA, V 978.2 \AA^{3}, Z=4$ ). The compound has a rather long $\mathrm{Pd}-\mathrm{CH}_{2}$ bond (2.096(1) $\AA$, mean).
\end{abstract}

\section{Introduction}

Metal sulfur ylide complexes were normally prepared in aprotic solvents [1]. In a previous report [2] we described the synthesis of sulfur ylide complexes by the phase transfer technique, a rarely used method [3]. Subsequently we found that some interesting metal complexes of sulfoxonium ylide could be prepared in aqueous solution [4]. Our continuing interest in metal ylide complexes, especially with the application of the phase transfer technique in their synthesis, has led us to study the reactions of these complexes, as made in our laboratory. One of the compounds studied in detail has been the iodo-bridged palladium dimer, $[\operatorname{Pd}(\mu$ I) $\left.\left(\left(\mathrm{CH}_{2}\right)_{2}(\mathrm{SO})\left(\mathrm{CH}_{3}\right)\right)\right]_{2}(1)$, which was obtained in almost quantitative yield by a simple technique developed in our laboratory. In this paper we also describe an $X$-ray diffraction study of $\left[\mathrm{Pd}\left(\left(\mathrm{CH}_{2}\right)_{2}(\mathrm{SO})\left(\mathrm{CH}_{3}\right)\right)_{2}\right]$ which contains two bidentate sulfoxonium ylides. 


\section{Experimental}

Infrared spectra were recorded on a Beckman Acculab TM1 spectrometer in $\mathrm{KBr}$ pellets. The ${ }^{1} \mathrm{H}$ NMR spectra were recorded on a Varian EM360 spectrometer. Chemical shifts are downfield relative to TMS standard. Melting points were measured on a Fisher-Johns Melting Point Apparatus. Elemental analyses were carried out by Taipei Instrumentation Center, Taiwan. The compounds 1, $\left.\left[\mathrm{PdI}\left(\left(\mathrm{CH}_{2}\right)(\mathrm{SO})\left(\mathrm{CH}_{3}\right)_{2}\right)\right)\left(\left(\mathrm{CH}_{2}\right)_{2}(\mathrm{SO})\left(\mathrm{CH}_{3}\right)\right)\right]$ (2), and $\left[\mathrm{Pd}\left(\left(\mathrm{CH}_{2}\right)_{2}(\mathrm{SO})\left(\mathrm{CH}_{3}\right)\right)-\right.$ $\left.\left(\left(\mathrm{CH}_{2}\right)(\mathrm{SO})\left(\mathrm{CH}_{3}\right)_{2}\right)_{2}\right] \mathrm{I}(3)$ were prepared by the methods developed in our laboratory [4]. $\left[\mathrm{Pd}\left(\left(\mathrm{CH}_{2}\right)_{2}(\mathrm{SO})\left(\mathrm{CH}_{3}\right)\right)_{2}\right](4)$ was prepared according to our previous work [4] but was modified slightly so that a better yield was obtained. Compound 1 (1.0 g, $1.5 \mathrm{mmol})$ and $\left(\left(\mathrm{CH}_{3}\right)_{3}(\mathrm{SO})\right) \mathrm{I}(0.75 \mathrm{~g}, 3.4 \mathrm{mmol})$ were dissolved in $4 \mathrm{ml}$ DMSO. To this yellow solution was added dropwise $5 \mathrm{~N}$ aqueous $\mathrm{NaOH}$ until the solution became colorless, this was then stirred overnight and $50 \mathrm{ml}$ of water was added. The yield was $53 \%$.

\section{$\left[\mathrm{Pd}\left(\left(\mathrm{CH}_{2}\right)_{2}(\mathrm{SO})\left(\mathrm{CH}_{3}\right)\right)\left(\mathrm{S}_{2} \mathrm{P}\left(\mathrm{OC}_{2} \mathrm{H}_{5}\right)_{2}\right)\right](5)$}

To compound $1\left(0.5 \mathrm{~g}, 7.7 \times 10^{-5} \mathrm{~mol}\right)$ suspended in $20 \mathrm{ml} \mathrm{CH}_{2} \mathrm{Cl}_{2}$ was added $\mathrm{NH}_{4} \mathrm{~S}_{2} \mathrm{P}\left(\mathrm{OC}_{2} \mathrm{H}_{5}\right)_{2}\left(0.034 \mathrm{~g}, 1.67 \times 10^{-4} \mathrm{~mol}\right)$. After stirring the mixture for $10 \mathrm{~min}$, the yellow suspension went into solution and some white solid was precipitated. The solution was stirred for a further hour, and then washed three times with water. The solvent was removed and the residue was recrystallized from $n$-hexane $/ \mathrm{CH}_{2} \mathrm{Cl}_{2}$. White needle-like crystals were obtained $(0.037 \mathrm{~g}, 63 \%$ yield $)$. When $\mathrm{n}-\mathrm{Bu}{ }_{4} \mathrm{NI}(0.01$ $\mathrm{g}, 2.7 \times 10^{-5} \mathrm{~mol}$ ) was added to the above reaction mixture, the yellow suspension dissolved within $5 \mathrm{~min}$. The final yield was $85 \%(0.05 \mathrm{~g})$; m.p. $88^{\circ} \mathrm{C}$. Anal. Found: C, 22.1; H, 4.4. $\mathrm{C}_{7} \mathrm{H}_{17} \mathrm{O}_{3} \mathrm{~S}_{3} \mathrm{Pd}$ calc: $\mathrm{C}, 22.0 ; \mathrm{H}, 4.5 \%$. IR $\nu(\mathrm{SO}) 1187 \mathrm{~cm}^{-1}{ }^{1} \mathrm{H}$ NMR 4.12(q of d, $\left.4 \mathrm{H},{ }^{2} J(\mathrm{H}, \mathrm{H}) 7 \mathrm{~Hz},{ }^{3} J(\mathrm{P}, \mathrm{H}) 10 \mathrm{~Hz}, \mathrm{OCH}_{2}\right), 3.45\left(\mathrm{t}, 3 \mathrm{H},{ }^{4} J(\mathrm{H}, \mathrm{H})\right.$ $\left.1.8 \mathrm{~Hz}, \mathrm{SCH}_{3}\right), 1.8-3.3\left(2\right.$ sets of $\left.\mathrm{m}, 4 \mathrm{H}, \mathrm{SCH}_{2}\right), 1.32\left(\mathrm{t}, 6 \mathrm{H},{ }^{2} \mathrm{~J}(\mathrm{H}, \mathrm{H}) 7 \mathrm{~Hz}, \mathrm{CH}_{3}\right)$ ppm.

$\left[\mathrm{Pd}\left(\left(\mathrm{CH}_{2}\right)_{2}(\mathrm{SO})\left(\mathrm{CH}_{3}\right)\right)\left(\mathrm{S}_{2} \mathrm{COC}_{2} \mathrm{H}_{5}\right)\right](6)$

This compound was prepared in a way similar to that for 5 . When no phase transfer catalyst (PTC) was added, the yellow solid 1 dissolved within $15 \mathrm{~min}$ and $0.039 \mathrm{~g}$ (79\% yield) of 6 was isolated. In the presence of $\mathrm{n}-\mathrm{Bu}_{4} \mathrm{NI}$, the solution went clear after $6 \mathrm{~min}$ and $0.044 \mathrm{~g}$ of 6 (90\% yield) was obtained. After recrystallization, light yellow needle-like crystals were obtained; m.p. $136^{\circ} \mathrm{C}$. Anal. Found: C, 22.8; $\mathrm{H}, 3.8 . \mathrm{C}_{6} \mathrm{H}_{12} \mathrm{O}_{2} \mathrm{~S}_{3} \mathrm{Pd}$ calc: $\mathrm{C}, 22.6 ; \mathrm{H}, 3.8 \%$. IR $\nu$ (SO) $1168 \mathrm{~cm}^{-1}$. ${ }^{1} \mathrm{H} \mathrm{NMR}$ $\left(\mathrm{CDCl}_{3}\right) 4.60\left(\mathrm{q}, 2 \mathrm{H},{ }^{2} J(\mathrm{H}, \mathrm{H}) 7 \mathrm{~Hz}, \mathrm{CH}_{2}\right), 3.43\left(\mathrm{t}, 3 \mathrm{H},{ }^{4} J(\mathrm{H}, \mathrm{H}) 1.8 \mathrm{~Hz}, \mathrm{SCH}_{3}\right)$, 1.87-3.24(2 sets $\left.\mathrm{m}, 4 \mathrm{H}, \mathrm{SCH}_{2}\right), 1.42\left(\mathrm{t}, 3 \mathrm{H},{ }^{2} \mathrm{~J}(\mathrm{H}, \mathrm{H}) 7 \mathrm{~Hz}, \mathrm{CH}_{3}\right) \mathrm{ppm}$.

\section{$\left[\mathrm{Pd}\left(\left(\mathrm{CH}_{2}\right)_{2}(\mathrm{SO})\left(\mathrm{CH}_{3}\right)\right)\left(\mathrm{S}_{2} \mathrm{CN}\left(\mathrm{C}_{2} \mathrm{H}_{5}\right)_{2}\right)\right]$ (7)}

This compound was prepared in a way similar that for 5 . Without PTC, a $56 \%$ yield $(0.030 \mathrm{~g})$ was obtained. In the presence of $\mathrm{n}-\mathrm{Bu}{ }_{4} \mathrm{NI}, 83 \%$ yield $(0.044 \mathrm{~g})$ was obtained. The compound forms golden needles after recrystallization; m.p. $115^{\circ} \mathrm{C}$. Anal. Found: $\mathrm{C}, 27.7 ; \mathrm{H}, 4.9 ; \mathrm{N}, 4.0 . \mathrm{C}_{8} \mathrm{H}_{17} \mathrm{NOS}_{3} \mathrm{Pd}$ calc: $\mathrm{C}, 27.8 ; \mathrm{H}, 4.9 ; \mathrm{N}, 4.1 \%$. IR $\nu$ (SO) $1175 \mathrm{~cm}^{-1} .{ }^{1} \mathrm{H}$ NMR $\left(\mathrm{CDCl}_{3}\right) 3.73\left(\mathrm{q}, 4 \mathrm{H},{ }^{2} J(\mathrm{H}, \mathrm{H}) 7 \mathrm{~Hz}, \mathrm{CH}_{2}\right), 3.46(\mathrm{t}$, $\left.3 \mathrm{H},{ }^{4} J(\mathrm{H}, \mathrm{H}) 1.8 \mathrm{~Hz}, \mathrm{SCH}_{3}\right) 1.87-3.13\left(2\right.$ sets $\left.\mathrm{m}, 4 \mathrm{H}, \mathrm{SCH}_{2}\right), 1.23\left(\mathrm{t}, 6 \mathrm{H},{ }^{2} J(\mathrm{H}, \mathrm{H}) 7\right.$ $\mathrm{Hz}, \mathrm{CH}_{3}$ ) ppm. 
$\left[\mathrm{Pd}\left(\left(\mathrm{CH}_{2}\right)_{2}(\mathrm{SO})\left(\mathrm{CH}_{3}\right)\right)(\mathrm{acac})\right](8)$

To compound $1\left(0.05 \mathrm{~g}, 7.7 \times 10^{-5} \mathrm{~mol}\right)$ and $0.02 \mathrm{ml} \mathrm{Hacac}\left(1.93 \times 10^{-4} \mathrm{~mol}\right)$ in $20 \mathrm{ml} \mathrm{CH} \mathrm{CH}_{2} \mathrm{Cl}_{2}$ was added $0.05 \mathrm{~N} \mathrm{NaOH}$ solution $(20 \mathrm{ml})$. The two-phase solution was stirred for $50 \mathrm{~min}$ before it went clear. After stirring for another $1 \mathrm{~h}$, the organic layer was separated and washed four times with water. The organic solution was then dried under vacuum. The pale yellow residue was washed with ether and recrystallized from $\mathrm{n}$-hexane $/ \mathrm{CH}_{2} \mathrm{Cl}_{2}$ yielding $0.033 \mathrm{~g}$ (72\% yield) of 8 . When 0.01 $\mathrm{g}\left(2.71 \times 10^{-5} \mathrm{~mol}\right)$ of $\mathrm{n}-\mathrm{Bu}_{4} \mathrm{NI}$ was added to the solution, it became clear within 10 min. Addition of 18-crown- 6 ether with $\mathrm{KOH}$ gave a similar result. The yields for the latter two reactions were $90 \%$ ( $0.04 \mathrm{~g})$; m.p. $128^{\circ} \mathrm{C}$ (decomposed). Anal. Found: $\mathrm{C}, 32.7 ; \mathrm{H}, 4.9 . \mathrm{C}_{8} \mathrm{H}_{14} \mathrm{O}_{3} \mathrm{SPd}$ calc: $\mathrm{C}, 32.4 ; \mathrm{H}, 4.7 \%$. IR $\nu(\mathrm{SO}) 1177 \mathrm{~cm}^{-1}$. ${ }^{1} \mathrm{H}$ NMR $\left(\mathrm{CDCl}_{3}\right) 5.3(\mathrm{~s}, 1 \mathrm{H}, \mathrm{CH}), 3.5\left(\mathrm{t}, 3 \mathrm{H},{ }^{4} J(\mathrm{H}, \mathrm{H}) 1.8 \mathrm{~Hz}, \mathrm{SCH}_{3}\right), 1.8-3.0(2$ sets m, $4 \mathrm{H}$, $\left.\mathrm{SCH}_{2}\right), 1.91\left(\mathrm{~s}, 6 \mathrm{H}, \mathrm{CCH}_{3}\right) \mathrm{ppm}$.

$\left[\mathrm{PdI}\left(\left(\mathrm{CH}_{2}\right)_{2}\left(\mathrm{SO}_{(}\right)\left(\mathrm{CH}_{3}\right)\right)\left(\mathrm{PPh}_{3}\right)\right]$ (9)

To compound $1\left(0.1 \mathrm{~g}, 1.54 \times 10^{-4} \mathrm{~mol}\right)$ in $20 \mathrm{ml} \mathrm{CH}_{2} \mathrm{Cl}_{2}$ was added $\mathrm{PPh}_{3}(0.08$ $\mathrm{g}, 3.08 \times 10^{-4} \mathrm{~mol}$ ). The resultant solution was stirred for $1 \mathrm{~h}$. The solvent was then removed in vacuum. The yellow residue was washed three times with ether. Recrystallization from $\mathrm{n}$-hexane $/ \mathrm{CH}_{2} \mathrm{Cl}_{2}$ gave yellow crystals $(0.64 \mathrm{~g}$ (91\% yield)), which were identified as compound 9 by comparison of its IR and ${ }^{1} \mathrm{H}$ NMR spectra, m.p. and elemental analysis with the authentic compound.

$\left[\mathrm{PdI}\left(\left(\mathrm{CH}_{2}\right)_{2}(\mathrm{SO})\left(\mathrm{CH}_{3}\right)\right)\left(\mathrm{P}(\mathrm{OMe})_{3}\right)\right](10)$

This compound was prepared by the method analogous to that for 9 , but was recrystallized from ethanol, yielding yellow needle-like crystals. Yield, 85\% (0.117 g); m.p. $132^{\circ} \mathrm{C}$ (decomposed). Anal. Found: C, 16.1; H, 3.6. $\mathrm{C}_{6} \mathrm{H}_{16} \mathrm{IO}_{4} \mathrm{PSPd}$ calc: $\mathrm{C}, 16.1 ; \mathrm{H}, 3.6 \%$. IR $\nu(\mathrm{SO}) 1202 \mathrm{~cm}^{-1}$. ${ }^{1} \mathrm{H}$ NMR $\left(\mathrm{CDCl}_{3}\right) 3.75\left(\mathrm{~d}, 9 \mathrm{H},{ }^{3} J(\mathrm{P}, \mathrm{H}) 12\right.$ $\left.\mathrm{Hz}, \mathrm{OCH}_{3}\right), 3.32\left(\mathrm{t}, 3 \mathrm{H},{ }^{4} \mathrm{~J}(\mathrm{H}, \mathrm{H}) 1.8 \mathrm{~Hz}, \mathrm{SCH}_{3}\right), 1.95-3.47\left(\mathrm{~m}, 4 \mathrm{H}, \mathrm{SCH}_{2}\right) \mathrm{ppm}$.

The following two compounds were prepared by the method similar to that described for 10.

$\left[\mathrm{PdI}\left(\left(\mathrm{CH}_{2}\right)_{2}(\mathrm{SO})\left(\mathrm{CH}_{3}\right)\right)\left(\mathrm{P}(\mathrm{OPh})_{3}\right)\right]$ (11)

Color, yellow. Yield $80 \%$; m.p. $139^{\circ} \mathrm{C}$ (decomposed). Anal. Found: C, 39.9; H, 3.3. $\mathrm{C}_{21} \mathrm{H}_{22} \mathrm{IO}_{4} \mathrm{PSPd}$ calc: $\mathrm{C}, 39.7 ; \mathrm{H}, 3.5 \%$. IR $\nu(\mathrm{SO}) 1205 \mathrm{~cm}^{-1}$. ${ }^{1} \mathrm{H}$ NMR $\left(\mathrm{CDCl}_{3}\right) 7.0-7.6\left(\mathrm{~m}, 30 \mathrm{H}, \mathrm{C}_{6} \mathrm{H}_{5}\right), 2.5\left(\mathrm{t}, 3 \mathrm{H},{ }^{4} \mathrm{~J}(\mathrm{H}, \mathrm{H}) 1.8 \mathrm{~Hz}, \mathrm{SCH}_{3}\right), 1.1-3.47(\mathrm{~m}$, $\left.4 \mathrm{H}, \mathrm{SCH}_{2}\right) \mathrm{ppm}$.

\section{$\left[\mathrm{PdI}\left(\left(\mathrm{CH}_{2}\right)_{2}(\mathrm{SO})\left(\mathrm{CH}_{3}\right)\right)\left(\mathrm{C}_{5} \mathrm{H}_{5} \mathrm{~N}\right)\right](12)$}

Color, yellow. Yield 86\%; m.p. $165^{\circ} \mathrm{C}$ (decomposed). Anal. Found: C, 23.9; H, 3.0; N, 3.6. $\mathrm{C}_{8} \mathrm{H}_{12}$ INOSPd calc: C, 23.8; H, 3.0; N, 3.5\%. IR $\nu(\mathrm{SO}) 1187 \mathrm{~cm}^{-1} .{ }^{1} \mathrm{H}$ NMR $\left(\mathrm{CDCl}_{3}\right) 7.3-8.9\left(\mathrm{~m}, 5 \mathrm{H}, \mathrm{C}_{5} \mathrm{H}_{5} \mathrm{~N}\right), 3.59\left(\mathrm{t}, 3 \mathrm{H},{ }^{4} J(\mathrm{H}, \mathrm{H}) 1.8 \mathrm{~Hz}, \mathrm{SCH}_{3}\right)$, 1.9-3.5(m, $\left.4 \mathrm{H}, \mathrm{SCH}_{2}\right) \mathrm{ppm}$.

\section{Reaction of $\left[\mathrm{Pd}\left(\left(\mathrm{CH}_{2}\right)_{2}(\mathrm{SO})\left(\mathrm{CH}_{3}\right)\right)_{2}\right]$ with $\mathrm{HI}$}

To compound $4\left(0.2 \mathrm{~g}, 6.9 \times 10^{-4} \mathrm{~mol}\right)$ in $20 \mathrm{ml} \mathrm{CHCl}_{3}$ was added dropwise to aqueous $\mathrm{HI}(0.09 \mathrm{ml}, 7.6 \mathrm{M})$, and immediately a yellow precipitation formed. After $1 \mathrm{~h}$ the solid was filtered and washed with water, alcohol, and then ether. The solid was identified as compound 1 by comparison of its IR, ${ }^{1} \mathrm{H}$ NMR spectra and m.p., with the authentic compound. 
Reaction of $\left[\mathrm{PdI}\left(\left(\mathrm{CH}_{2}\right)\left(\mathrm{SO}_{2}\left(\mathrm{CH}_{3}\right)_{2}\right)\left(\left(\mathrm{CH}_{2}\right)_{2}(\mathrm{SO})\left(\mathrm{CH}_{3}\right)\right)\right]\right.$ and $\left[\mathrm{Pd}\left(\left(\mathrm{CH}_{2}\right)_{2}^{-}\right.\right.$ ( $\left.\left.\mathrm{SO})\left(\mathrm{CH}_{3}\right)\right)\left(\left(\mathrm{CH}_{2}\right)(\mathrm{SO})\left(\mathrm{CH}_{3}\right)_{2}\right)_{2}\right] I$ with $\mathrm{HI}$

These two reactions were carried out under the same conditions as the previous experiment, except that the solvent used in this case was DMSO. The products isolated were identified as $\mathbf{1}$.

$\left[\mathrm{Pd}(\mu-\mathrm{Cl})\left(\left(\mathrm{CH}_{2}\right)_{2}(\mathrm{SO})\left(\mathrm{CH}_{3}\right)\right)\right]_{2}(\mathrm{I3})$

Compound $4\left(0.1 \mathrm{~g}, 3.5 \times 10^{-4} \mathrm{~mol}\right)$ in $20 \mathrm{ml} \mathrm{CHCl}$ was treated with $\mathrm{HCl}(12$ $M, 0.064 \mathrm{ml}$ ), after stirring for $20 \mathrm{~min}$, some yellow solid was separated. Stirring was continued for a further $24 \mathrm{~h}$. The solid was then filtered and washed with $\mathrm{H}_{2} \mathrm{O}$. Recrystallization from DMSO $/ \mathrm{H}_{2} \mathrm{O}$ gave needle-like crystals. Yield 50\%. Anal. Found: C, 15.6; H, 3.1. $\mathrm{C}_{6} \mathrm{H}_{14} \mathrm{O}_{2} \mathrm{~S}_{2} \mathrm{Cl}_{2} \mathrm{Pd}_{2}$ calc: C, 15.5; H, 3.9\%. IR $\nu$ (CO) 1192 $\mathrm{cm}^{-1} .{ }^{1} \mathrm{H}$ NMR (DMSO- $\left.d_{6}\right) 3.53\left(\mathrm{t}, 3 \mathrm{H},{ }^{4} \mathrm{~J}(\mathrm{H}, \mathrm{H}) 1.8 \mathrm{~Hz}, \mathrm{SCH}_{3}\right) 1.83-3.23(\mathrm{~m}, 4 \mathrm{H}$, $\left.\mathrm{SCH}_{2}\right)$ ppm.

Reaction of $\left[\mathrm{Pd}\left(\left(\mathrm{CH}_{2}\right)_{2}(\mathrm{SO})\left(\mathrm{CH}_{3}\right)\right)_{2}\right]$ with $\mathrm{I}_{2}$

To a solution of compound $4\left(0.29 \mathrm{~g}, 6.9 \times 10^{-4} \mathrm{~mol}\right)$ in $30 \mathrm{ml} \mathrm{CHCl}_{3}$, was added $\mathrm{I}_{2}\left(0.359 \mathrm{~g}, 1.4 \times 10^{-3} \mathrm{~mol}\right)$ in $15 \mathrm{ml} \mathrm{CCl}_{4}$. After $5 \mathrm{~min}$ the solution turned to golden yellow and a yellow solid precipitated. Stirring was continued for $30 \mathrm{~min}$. The solid was filtered and washed with $\mathrm{CHCl}_{3}$. The yellow solid was formulated to be $\mathrm{Pd}\left(\left(\mathrm{CH}_{2}\right)_{2}(\mathrm{SO})\left(\mathrm{CH}_{3}\right)\right)_{2} \mathrm{I}_{2}$ (Found: $\mathrm{C}, 13.8 ; \mathrm{H}, 2.6 . \mathrm{C}_{6} \mathrm{H}_{14} \mathrm{O}_{2} \mathrm{I}_{2} \mathrm{~S}_{2} \mathrm{Pd}$ calc: $\mathrm{C}$, $13.3 ; \mathrm{H}, 2.6 \%)$ It decomposed in air within $2 \mathrm{~h}$. It decomposed immediately in DMSO solution. The compounds isolated from both the filtrate and the decomposed product were found to be compound 1 .

Reaction of $\left[\mathrm{PdI}\left(\left(\mathrm{CH}_{2}\right)(\mathrm{SO})\left(\mathrm{CH}_{3}\right)_{2}\right)\left(\left(\mathrm{CH}_{2}\right)_{2}(\mathrm{SO})\left(\mathrm{CH}_{3}\right)\right)\right]$ with $\mathrm{NaOH}$

To compound $2\left(0.2 \mathrm{~g}, 4.8 \times 10^{-4} \mathrm{~mol}\right)$ in $3 \mathrm{ml}$ DMSO was added dropwise $5 \mathrm{~N}$ $\mathrm{NaOH} 0.05 \mathrm{ml}$. The resultant solution was set aside for $2 \mathrm{~d}$. The solid thus obtained was found to be compound 1 .

Crystal data for $\left[\mathrm{Pd}\left(\left(\mathrm{CH}_{2}\right)_{2}\left(\mathrm{SO}_{1}\left(\mathrm{CH}_{3}\right)\right)_{2}\right]\right.$

$\left[\mathrm{Pd}\left(\left(\mathrm{CH}_{2}\right)_{2}(\mathrm{SO})\left(\mathrm{CH}_{3}\right)\right)_{2}\right]$, mol. wt. 288.4, orthorhombic, Pbcn, a 13.379(2), b 8.081(1), c 9.048(2) $\AA, V 978.2 \AA^{3}, Z=4, D_{\mathrm{c}} 1.96 \mathrm{~g} / \mathrm{cm}^{3}, D_{\mathrm{m}} 1.92 \mathrm{~g} / \mathrm{cm}^{3}$, $\lambda\left(\mathrm{Mo}-K_{\alpha}\right)=0.7093 \AA$, $\mu 22.38 \mathrm{~cm}^{-1} . F(000)=576$.

\section{Collection and reduction of $X$-ray data}

A black crystal of dimensions $0.2 \times 0.4 \times 0.7 \mathrm{~mm}$ was used for data collection. Diffraction data were collected on a CAD4 diffractometer using graphite-monochromated $\mathrm{Mo}-K_{\alpha}$ radiation. Unit cell dimensions were determined from a least squares refinement of 24 carefully centered reflections $\left(27.98<2 \theta<40.88^{\circ}\right)$. Intensity data within $2 \theta<70^{\circ}$ were collected at scan speeds between $20 / 3$ to $20 / 23$ $\mathrm{deg} / \mathrm{min}$ by use of $\omega-2 \theta$ scan techniques, and a scan range calculated from the expression $\Delta \theta=2(0.7+0.35 \tan \theta)^{\circ}$. Stationary background measurements were taken before and after each scan for a time equal to quarter of the scan time. Variation of three standard reflections monitored every two hours $<3 \%$. After correction for background, the intensities were reduced to $F$ and $\sigma(F)$ from counting statistics, and weights were assigned as $w=1 / \sigma^{2}(F)$. Of 2469 total reflections, 1294 having $I>2.5 \sigma(I)$ were considered observed. Intensity data were 
Table 1

Positional and thermal parameters ${ }^{a}$ of $\left[\operatorname{Pd}\left(\left(\mathrm{CH}_{2}\right)_{2} \mathrm{SO}\left(\mathrm{CH}_{3}\right)\right)_{2}\right]$

\begin{tabular}{|c|c|c|c|c|c|c|}
\hline Atom & $x$ & $y$ & $z$ & $B_{\text {iso }}$ & & \\
\hline$\overline{\mathrm{Pd}}$ & 0.00000 & 0.00000 & 0.00000 & $2.02(1)$ & & \\
\hline $\mathbf{S}$ & $0.11172(2)$ & $0.27266(3)$ & $0.05860(3)$ & $2.31(1)$ & & \\
\hline O & $0.1448(1)$ & $0.4126(1)$ & $0.1469(1)$ & $3.37(5)$ & & \\
\hline $\mathrm{C}(1)$ & $-0.0114(1)$ & $0.2590(1)$ & $0.0048(2)$ & $2.69(5)$ & & \\
\hline$C(2)$ & $-0.1221(1)$ & $-0.0764(1)$ & $-0.1274(2)$ & $2.46(6)$ & & \\
\hline$C(3)$ & $0.1813(2)$ & $0.2716(2)$ & $-0.1079(2)$ & $4.03(9)$ & & \\
\hline $\mathbf{H}(11)$ & $-0.0490(7)$ & $0.2974(14)$ & $0.0804(10)$ & $2.9(3)$ & & \\
\hline$H(12)$ & $-0.0273(8)$ & $0.3358(13)$ & $-0.0707(10)$ & $4.2(3)$ & & \\
\hline $\mathbf{H}(21)$ & $-0.0974(8)$ & $-0.0663(10)$ & $-0.2120(9)$ & $1.5(2)$ & & \\
\hline$H(22)$ & $-0.1825(7)$ & $-0.0388(11)$ & $-0.1232(11)$ & $2.0(2)$ & & \\
\hline$H(31)$ & $0.1727(11)$ & $0.3552(17)$ & $0.8523(16)$ & $5.2(5)$ & & \\
\hline $\mathrm{H}(32)$ & $0.2430(11)$ & $0.2597(19)$ & $-0.0793(15)$ & $7.7(5)$ & & \\
\hline $\mathrm{H}(33)$ & $0.1741(14)$ & $0.1766(25)$ & $-0.1838(19)$ & $13.8(7)$ & & \\
\hline Atom & $U_{11}$ & $U_{22}$ & $U_{33}$ & $U_{12}$ & $U_{13}$ & $U_{23}$ \\
\hline Pd & $2.52(1)$ & $2.21(1)$ & $2.96(1)$ & $-0.16(1)$ & $-0.14(1)$ & $0.14(1)$ \\
\hline $\mathbf{S}$ & $2.86(2)$ & $2.56(1)$ & $3.34(2)$ & $-0.34(1)$ & $0.06(1)$ & $-0.24(1)$ \\
\hline 0 & $4.39(5)$ & $3.11(4)$ & $5.28(6)$ & $-0.71(4)$ & $-0.54(5)$ & $-1.11(4)$ \\
\hline $\mathrm{C}(1)$ & $3.07(7)$ & $2.72(5)$ & $4.45(6)$ & $0.20(6)$ & $-0.14(8)$ & $0.24(8)$ \\
\hline $\mathrm{C}(2)$ & $2.72(7)$ & $3.21(6)$ & $3.41(8)$ & $0.26(6)$ & $-0.23(7)$ & $0.12(6)$ \\
\hline$C(3)$ & $5.50(11)$ & $4.95(10)$ & $4.86(11)$ & $-0.90(9)$ & $2.10(9)$ & $0.42(9)$ \\
\hline
\end{tabular}

a Temperature factors $=-2 \pi^{2}\left(U_{11} h h a^{\star} a^{\star}+\ldots+2 U_{12} h k a^{\star} b^{\star}+\ldots\right)$, the $U_{i j}$ values are $\times 100$.

\section{TABLE 2}

Bond distances $(\stackrel{\AA}{\mathrm{A}})$ and angles $\left(^{\circ}\right)$ of $\left[\mathrm{Pd}\left(\left(\mathrm{CH}_{2}\right)_{2} \mathrm{SO}\left(\mathrm{CH}_{3}\right)\right)_{2}\right]$

\begin{tabular}{|c|c|c|c|c|c|}
\hline $\mathrm{Pd}-\mathrm{S}$ & $2.7148(3)$ & $\mathrm{Pd}-\mathrm{C}(1)$ & $2.099(1)$ & $P d-C(2)$ & $2.093(1)$ \\
\hline $\mathrm{Pd}-\mathrm{C}(2)$ & $2.093(1)$ & S-O & $1.453(1)$ & $\mathbf{S}-\mathbf{C}(\mathbf{1})$ & $1.722(1)$ \\
\hline$S-C(2)$ & $1.709(1)$ & S-C(3) & $1.771(1)$ & $\mathrm{C}(1)-\mathrm{H}(11)$ & $0.904(9)$ \\
\hline $\mathrm{C}(1)-\mathrm{H}(12)$ & $0.947(9)$ & $\mathrm{C}(2)-\mathrm{H}(21)$ & $0.838(8)$ & $\mathrm{C}(2)-\mathrm{H}(22)$ & $0.864(9)$ \\
\hline$C(3)-H(31)$ & $0.77(1)$ & $\mathrm{C}(3)-\mathrm{H}(32)$ & $0.87(1)$ & $C(3)-H(33)$ & $1.03(2)$ \\
\hline$C(1)-P d-C(1)$ & & $180.00(0)$ & $\mathrm{C}(1)-\mathrm{Pd}-\mathrm{C}(2)$ & \multicolumn{2}{|c|}{$104.40(4)$} \\
\hline$C(1)-P d-C(2)$ & & $75.60(4)$ & $C(1)-P d-C(2)$ & \multicolumn{2}{|c|}{$75.60(4)$} \\
\hline$C(1)-P d-C(2)$ & & $104.40(4)$ & $\mathrm{C}(2)-P d-C(2)$ & \multicolumn{2}{|c|}{$180.00(0)$} \\
\hline $\mathrm{O}-\mathrm{S}-\mathrm{C}(1)$ & & $119.79(5)$ & O-S-C(2) & \multicolumn{2}{|c|}{$119.78(5)$} \\
\hline $\mathrm{O}-\mathrm{S}-\mathrm{C}(3)$ & & $108.13(7)$ & $C(1)-S-C(2)$ & \multicolumn{2}{|c|}{$96.97(5)$} \\
\hline$C(1)-S-C(3)$ & & $105.21(8)$ & $C(2)-S-C(3)$ & \multicolumn{2}{|c|}{$105.22(8)$} \\
\hline$P d-C(1)-S$ & & $90.01(5)$ & $\mathrm{Pd}-\mathrm{C}(1)-\mathrm{H}(11)$ & \multicolumn{2}{|c|}{$113.5(6)$} \\
\hline $\mathrm{Pd}-\mathrm{C}(1)-\mathrm{H}(12)$ & & $130.9(6)$ & $S-C(1)-H(11)$ & \multicolumn{2}{|c|}{$107.2(6)$} \\
\hline S-C(1)-H(12) & & $112.1(6)$ & $\mathrm{H}(11)-\mathrm{C}(1)-\mathrm{H}(12)$ & \multicolumn{2}{|c|}{$101.3(9)$} \\
\hline$P d-C(2)-S$ & & $90.54(5)$ & $P d-C(2)-H(21)$ & \multicolumn{2}{|c|}{$99.6(6)$} \\
\hline $\mathrm{Pd}-\mathrm{C}(2)-\mathrm{H}(22)$ & & $127.0(6)$ & $S-C(2)-H(21)$ & \multicolumn{2}{|c|}{$113.0(5)$} \\
\hline S-C(2)-H(22) & & $112.7(5)$ & $\mathrm{H}(21)-\mathrm{C}(2)-\mathrm{H}(22)$ & \multicolumn{2}{|c|}{$112.0(9)$} \\
\hline$S-C(3)-H(31)$ & & $108(1)$ & $S-C(3)-H(32)$ & \multicolumn{2}{|c|}{$104.3(8)$} \\
\hline S-C(3)-H(33) & & $121(1)$ & $H(31)-C(3)-H(32)$ & \multicolumn{2}{|c|}{$112(1)$} \\
\hline$H(31)-C(3)-H(33)$ & & $109(1)$ & $H(32)-C(3)-H(33)$ & \multicolumn{2}{|c|}{$102(1)$} \\
\hline
\end{tabular}


corrected for Lorentz-polarization effects and absorption by experimental $\psi$ rotation [11].

\section{Solution and refinement of structure}

All calculations were carried out with the NRCC SDP PDP-11 package [5], and ORTEP from Enraf-Nonius structure determination package [6] on a PDP-11/23 computer. The palladium atom was located from Patterson map, and subsequent heavy-atom Fourier synthesis revealed the position of the non-hydrogen atoms of the complex. The $\mathrm{H}$ atoms were located from difference Fourier map. The final least-squares cycle with anisotropic thermal parameters for all non-hydrogen atoms and isotropic parameters for hydrogen atoms gave $R$ and $R_{\mathrm{w}}$ factors of $5.6 \%$ and $2.2 \%$ respectively. The positional and thermal parameters of all the atoms are given in Table 1. The bond distances and angles are listed in Table 2. Tables of structure factors may be obtained from the authors.

\section{Results and discussion}

Reaction of 1 with soft chelating ligands such as sodium 1,1-dithiolates gave stable mixed-ligand compounds (5-7) which contain a bidentate sulfoxonium ylide and a bidentate 1,1-dithiolate ligand. All reactions proceeded very smoothly in chloroform solution. The reaction of 1 with $\mathrm{NaS}_{2} \mathrm{COEt}\left(\mathrm{Et}=\mathrm{C}_{2} \mathrm{H}_{5}\right)$ was followed by ${ }^{1} \mathrm{H}$ NMR spectroscopy. Without PTC, the ratio of complexed to free $\mathrm{NaS}_{2} \mathrm{COEt}$ was 8.3 after $2 \mathrm{~h}$. In the presence of $\mathrm{n}-\mathrm{Bu}_{4} \mathrm{NI}\left(10 \%\right.$ molar ratio of $\left.\mathrm{NaS}_{2} \mathrm{COEt}\right)$, the ratio of complexed to uncomplexed $\mathrm{NaS}_{2} \mathrm{COEt}$ was 12.6 (after $2 \mathrm{~h}$ ). Prolonged reaction did not increase the yield. An analogous compound, $\left[\mathrm{Ni}\left(\left(\mathrm{CH}_{2}\right)_{2}(\mathrm{SO})\right.\right.$ $\left.\left.\left(\mathrm{CH}_{3}\right)\right)(\mathrm{SacSac})\right]$, reported by Dudis et al. was prepared through a different route [7]. The reaction of 1 with diketone under basic conditions gave 8. Although PTC is not required for the above reaction, addition of PTC increased the reaction rate drastically. To our surprise, 1 did not react with oxalate anion, not even in the presence of PTC in various solvent systems. When 1 was treated with bases such as phosphine or pyridine, compounds formulated as $\left[\mathrm{PdI}\left(\left(\mathrm{CH}_{2}\right)_{2}(\mathrm{SO})\left(\mathrm{CH}_{3}\right)\right)(\right.$ base $\left.)\right]$ (9-12) were obtained. One compound, $\left[\mathrm{PdI}\left(\left(\mathrm{CH}_{2}\right)_{2}(\mathrm{SO})\left(\mathrm{CH}_{3}\right)\right)\left(\mathrm{PPh}_{3}\right)\right]$, which has been reported previously by us [2], was prepared by phase transfer catalyzed reaction of $\left.\left[\mathrm{PdCl}_{2}\left(\mathrm{PPh}_{3}\right)_{2}\right)\right]$ with $\left(\left(\mathrm{CH}_{3}\right)_{3}(\mathrm{SO})\right) \mathrm{I}$ and $\mathrm{NaOH}$ (Scheme 1).

Fackler et al. [8] suggested that a chelating ylide might have the ability to stabilize the high oxidation state of metals. Thus we attempted the oxidation of 4 with $\mathrm{CH}_{3} \mathrm{I}$ and $\mathrm{I}_{2}$. Compound 4 was found to be unreactive toward $\mathrm{CH}_{3} \mathrm{I}$ but reactive toward $I_{2}$. After reaction of 4 with $I_{2}$, we isolated an unstable yellow compound and the pale yellow compound 1 . The former slowly turned to brown in air. The final decomposed product was found to be 1 .

We have also carried out the reaction of $\mathrm{HI}$ and $\mathrm{HCl}$ with various sulfur ylide compounds such as 2,3 , and 4 . All the reactions with $\mathrm{HI}$ gave 1 , however, reaction of 4 with $\mathrm{HCl}$ gave a new compound $\left[\mathrm{Pd}(\mu-\mathrm{Cl})\left(\left(\mathrm{CH}_{2}\right)_{2}(\mathrm{SO})\left(\mathrm{CH}_{3}\right)\right)\right]_{2}(13)$, which is the chloro-analog of 1 . Attempts to prepare 13 directly by treating $\left(\mathrm{NH}_{4}\right)_{2} \mathrm{PdCl}_{4}$ with $\left(\left(\mathrm{CH}_{3}\right)_{3}(\mathrm{SO})\right) \mathrm{Cl}$ and $\mathrm{NaOH}$ under various conditions were unsuccessful. In all cases black metallic powder was formed. In one case a small amount of 13 was obtained. 


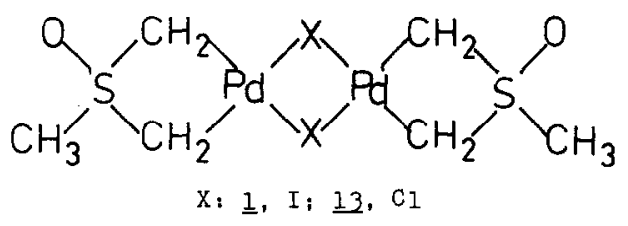

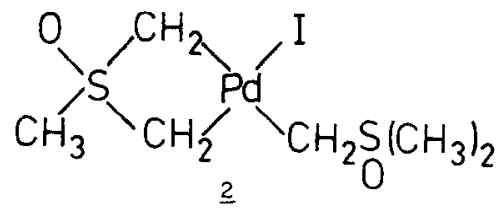

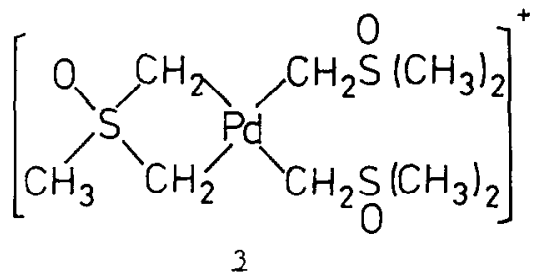<smiles>CC1(C[SH](C)(=O)O)CP(CS(C)(C)O)C1</smiles><smiles>CS1(O)CS[PH]2(CSCS2)C1</smiles>

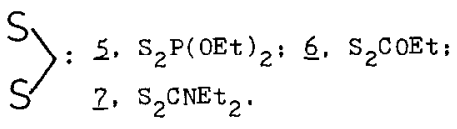<smiles>CC1OP2CC(C)(O1)OC2C</smiles>

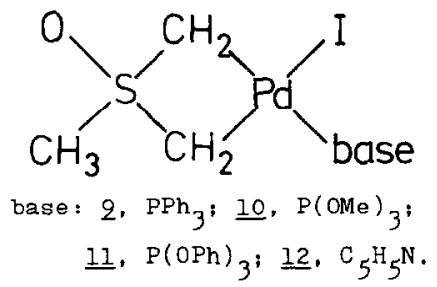

Scheme 1

It occurred to us that 2 is both a bidentate and a monodentate sulfoxonium ylide, thus removal of a methyl proton from the monodentate ylide should convert 2 to 4 . In our hands the reaction of $\mathrm{NaOH}$ with 2, however, gave 1 instead of 4 .

Thus treating $\mathrm{HX}$ and $\mathrm{NaOH}$ with various sulfoxonium ylide compounds suggest that the monodentate sulfoxonium ylide is unstable toward both acids and bases.

\section{Crystal and molecular structure}

The structural study of $\left[\mathrm{Pd}\left(\left(\mathrm{CH}_{2}\right)_{2}(\mathrm{SO})\left(\mathrm{CH}_{3}\right)\right)_{2}\right]$ presents the first example of a homoleptic complex containing two chelated sulfoxonium double-ylides (Fig. 1). The geometry about the palladium atom is distorted square-planar. The molecule has a center of inversion about the palladium atom. The four-membered ring formed by the chelated double ylide is puckered (Fig. 2). The dihedral angle between the $\mathrm{C}(1)-\mathrm{Pd}-\mathrm{C}(2)$ plane and the $\mathrm{C}(1)-\mathrm{S}(1)-\mathrm{C}(2)$ plane is $22.19^{\circ}$. The sulfur 


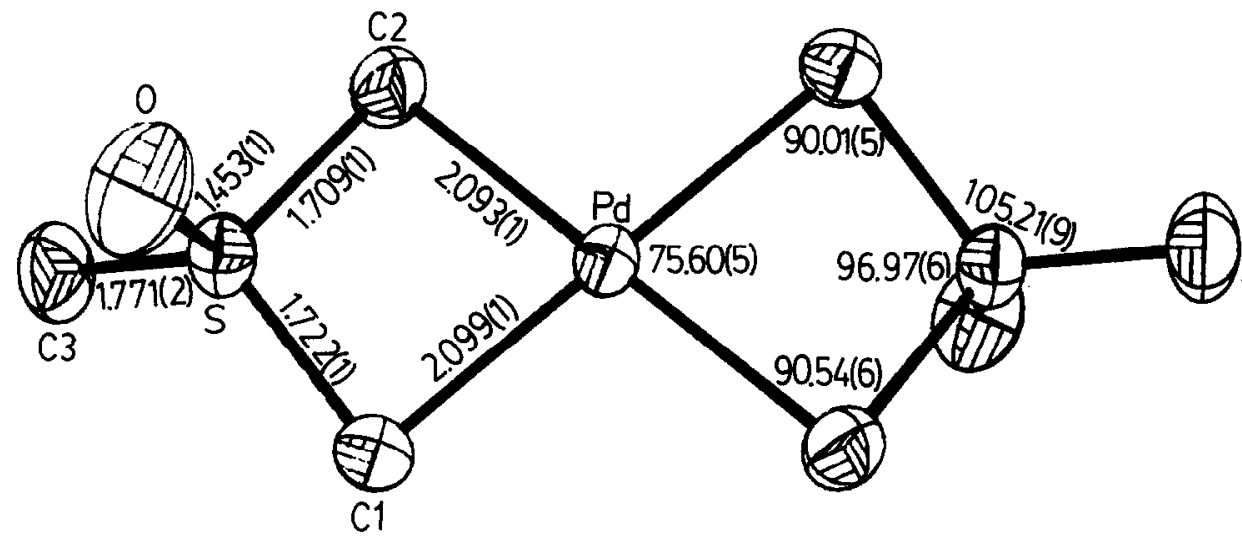

Fig. 1. ORTEP drawing of $\left[\operatorname{Pd}\left(\left(\mathrm{CH}_{2}\right)_{2}(\mathrm{SO})\left(\mathrm{CH}_{3}\right)\right)_{2}\right]$ with labelling scheme and selected bond parameters.

atom is $0.53 \AA$ above the $\mathrm{C}(1)-\mathrm{Pd}-\mathrm{C}(2)$ plane. This value is very close to the $0.55 \AA$ found in $\left[\mathrm{Pd}\left(\left(\mathrm{CH}_{2}\right)_{2}(\mathrm{SO})\left(\mathrm{CH}_{3}\right)\right)\left(\left(\mathrm{CH}_{2}\right)(\mathrm{SO})\left(\mathrm{CH}_{3}\right)_{2}\right)_{2}\right] \mathrm{I}$ [4]. The two $\mathrm{CH}_{3}$ groups are in the axial positions, such that the compound adopts the chair conformation.

Two geometric isomers are possible for this compound. One isomer with two oxygen atoms in the cis position and the other with two oxygen atoms in a trans position. The structure we arrived at is a trans isomer which is required for centrosymmetric geometry. When this compound was freshly precipitated from DMSO only one set of ${ }^{1} \mathrm{H}$ NMR signals was observed in $\mathrm{CDCl}_{3}$. The IR spectrum of the freshly precipitated solid and that of the solid obtained after recrystallization were identical. Thus the trans isomer was obtained directly from the reaction

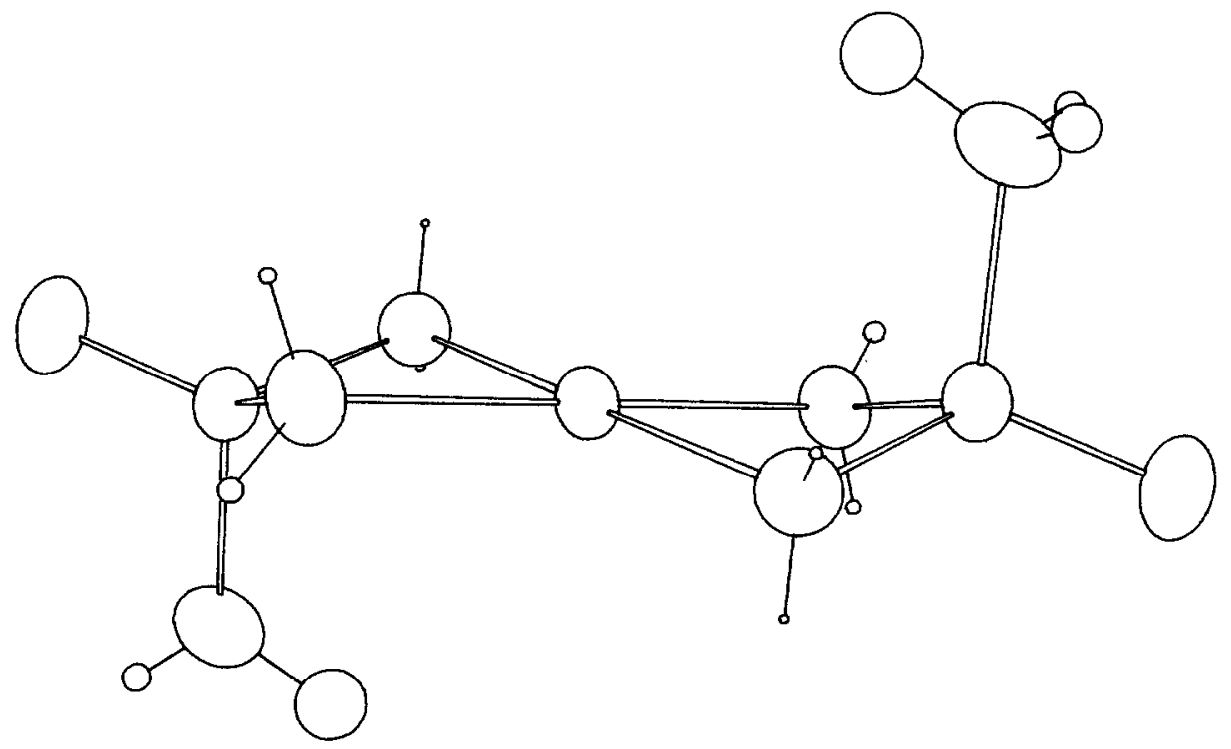

Fig. 2. Different view of $\left[\mathrm{Pd}\left(\left(\mathrm{CH}_{2}\right)_{2}(\mathrm{SO})\left(\mathrm{CH}_{3}\right)\right)_{2}\right]$ illustrating the puckered conformation. 
solution and not from the isomerization of $c i s$ isomer. In spite of the data we were unable to justify the reason for the trans configuration, since the trans isomer seems the more sterically favored.

In this compound the $\mathrm{S}-\mathrm{C}(3)$ distance $(1.771(2) \AA)$ is longer than the ylide type $\mathrm{S}-\mathrm{CH}_{2}$ distances $(1.721(1), 1.709(1) \AA)$, but is slightly shorter than ordinary S-C bond lengths found in organic sulfonium salts $(1.78-1.84 \AA$ ) [9]. The averaged $\mathrm{Pd}-\mathrm{CH}_{2}$ distance $(2.096(1) \AA)$ is longer than that found in $\left[\mathrm{Pd}\left(\mathrm{CH}_{2} \mathrm{C}\right.\right.$ $\left.\left.\left(=\mathrm{NNMe}_{2}\right) \mathrm{C}\left(\mathrm{CH}_{3}\right)_{3}\right)(\mathrm{acac})\right]^{+}(1.991 \AA)[10]$. Thus the structural results of our compound suggest that the ylidic character is partly retained upon complexation and that the metal-ylide bond might be weaker than normal metal-carbon bonds.

\section{Acknowledgement}

The authors would like to express their appreciation to the National Science Council for support of this work both for the research grant and for the use of the CAD-4 diffractometer.

\section{References}

1 L. Weber, Angew. Chem. Int. Ed. Engl., 22 (1983) 516.

2 I.J.B. Lin, H.C. Lai, S.C. Wu and L. Hwan, J. Organomet. Chem., 306 (1986) C24.

3 (a) H. Alper, Adv. Organomet. Chem., 19 (1981) 183; (b) G. Tanguy, J.C. Clement and H. Des Abbayes, J. Organomet. Chem., 314 (1986) C43.

4 I.J.B. Lin, L. Hwan, H.C. Shy, M.C. Cheng and Y. Wang, J. Organomet. Chem., 315 (1986) 135.

5 E.J. Gabe and F.L. Lee, Acta Cryst., A37, (1981) S339.

6 Enraf-Nonius, Structure Determination Package, (1979), Enraf-Nonius, Delft.

7 D.S. Dudis and J.P. Fackler, Jr., J. Organomet. Chem., 249 (1983) 289.

8 J.P. Fackler Jr. and C.J. Paparizos, J. Am. Chem. Soc., 99 (1977) 2363.

9 E.F. Perozzi and I.C. Paul, in C.J.M. Stirling, and S. Patai, (Eds.) The Chemistry of the Sulfonium Group; Wiley, New York, 1981, Part 1, pp. 1577.

10 A.G. Constable, W.S. McDonald, L.C. Sawkins and B.L. Shaw, J. Chem. Soc., Dalton Trans., (1980) 1992.

11 A.C.T. North, D.C. Phillips and F.S. Mathews, Acta Cryst., Scct. A, 24 (1968) 351. 